\title{
Éditorial
}

\section{Le classement de Shanghaï : encore un produit chinois de mauvaise qualité ?}

\author{
Jacky Samson*
}

Ce n'est pas ceux qui savent le mieux parler qui ont les meilleures choses à dire. Proverbe chinois

En 1998, lors du $100^{\mathrm{e}}$ anniversaire de l'Université de Pékin, le Président chinois a souhaité la mise en place d'un système pour évaluer le niveau de la recherche dans les universités chinoises ; ceci dans le but d'aider les universités chinoises à progresser. Le gouvernement chinois a désigné neuf universités pour réaliser ce projet. C'est l'Institute of Higher Education of the Jiao Tong University de Shanghaï, sous la direction du Professeur Nian Cai Liu, qui a présenté le projet le plus abouti. Et pour répondre à la demande de ses collègues, il a publié ce document interne sur internet en 2003, sous le titre Academic Ranking of World Universities (ARWU). Sous ce terme sans ambiguïté, on trouvait le classement des 500 meilleures universités mondiale sur les 17000 répertoriées. Chaque année (cette année le 15 août), cet institut effectue une mise à jour de ce classement, plus connu en France sous le nom de classement de Shanghaï. Depuis toujours, les premières places de ce classement reviennent aux universités américaines; en 2013 [1], elles occupent 17 des 20 premières places et la première université française (Université Pierre et Marie Curie Paris VI) arrive en $37^{\mathrm{e}}$ position. On retrouve 3 autres universités françaises dans les 100 premières places: l'Université Paris Sud - Paris XI à la $39^{\mathrm{e}}$ place, l'École Normale Supérieure de Paris à la $71^{\mathrm{e}}$ et l'Université de Strasbourg à la $97^{\mathrm{e}}$. Cette dernière qui avait quitté le Top 100 depuis 2008, le réintègre principalement grâce à l'un de ses chercheurs, Jules Hoffmann, qui a obtenu le prix Nobel de physiologie-médecine en 2011 ; elle ne l'a pas réintégré en 2012 car le classement de Shanghaï avait oublié de prendre cette distinction en compte. Il n'y a pas eu d'importantes variations au cours des années dans les 200 premières places; toutefois, on doit noter le parcours spectaculaire de l'Université de Shanghaï qui est passée de la catégorie $401^{\mathrm{e}}-500^{\mathrm{e}}$ places à la catégorie $151^{\mathrm{e}}-200^{\mathrm{e}}$ places (après la $100^{\mathrm{e}}$ place, les universités sont classées par groupes de 50 entre 101 et 200, puis par groupes de 100 entre 201 et 500).

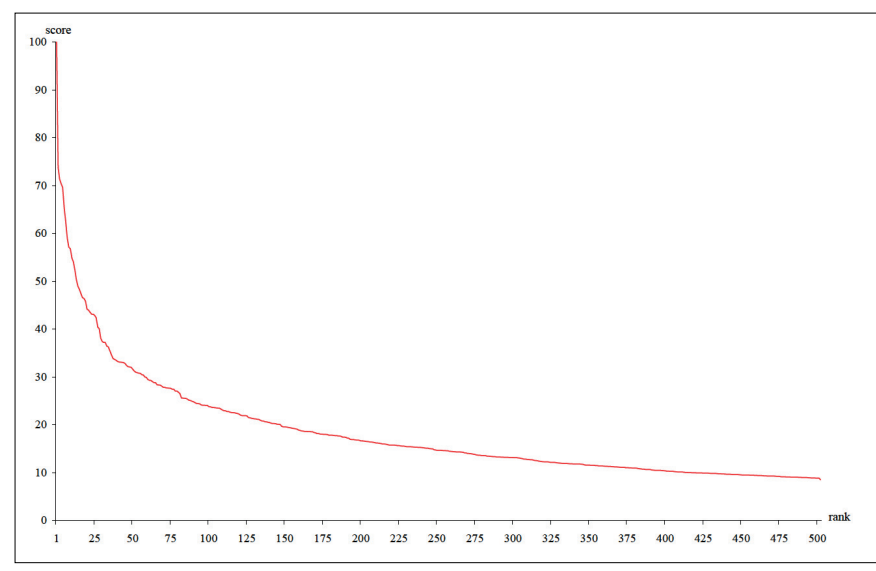

Fig. 1. Distribution des scores normalisés des 500 meilleures universités mondiales d'après le classement de Sanghaï 2008 [2]. Fig. 1. Distribution of normalized scores for the 500 best universities in the Shanghai ranking in 2008 [2].

Quand on examine la courbe de distribution des scores des 500 meilleures universités (Fig. 1), on comprend aisément pourquoi il est impossible d'attribuer une place précise après la $100^{\mathrm{e}}$ place avec une courbe aussi plate. Et devant une courbe aussi plate, on comprend aussi que la marge d'erreur est importante et que les approximations dans le calcul des scores réduisent ce classement à un simulacre.

Chaque année, la publication de ce classement soulève un tollé et, en France, le ministre de l'Enseignement supérieur et de la Recherche se sent obligé d'intervenir, reprenant toujours à peu près la même litanie. En 2013, Geneviève Fioraso a rappelé dans un communiqué que « les critères utilisés sont bien davantage adaptés aux universités anglo-saxonnes qu'aux universités européennes ", que "l'accent est mis sur un faible nombre d'universités, les universités de recherche, sans prise

\footnotetext{
*Correspondance : jacky.samson@unige.ch
} 
Tableau I. Critères utilisés pour le classement de Shanghaï. Table I. Criteria used for Academic Ranking of World Universities.

\begin{tabular}{|l|l|c|c|}
\hline \multicolumn{1}{|c|}{ Domaines } & \multicolumn{1}{|c|}{ Critères } & Pondération & Période \\
\hline $\begin{array}{l}\text { Qualité de } \\
\text { l'enseignement }\end{array}$ & $\begin{array}{l}\text { Nb prix Nobel et médailles } \\
\text { Fields / anciens élèves }\end{array}$ & $10 \%$ & 100 ans \\
\hline \multirow{2}{*}{$\begin{array}{l}\text { Qualité de } \\
\text { l'institution }\end{array}$} & $\begin{array}{l}\text { Nb prix Nobel et médailles } \\
\text { Fields / chercheurs }\end{array}$ & $20 \%$ & 100 ans \\
\cline { 2 - 4 } & $\begin{array}{l}\text { Nb chercheurs les plus } \\
\text { cités dans leur discipline }\end{array}$ & $20 \%$ & 20 ans \\
\hline \multirow{2}{*}{ Publications } & $\begin{array}{l}\text { Articles publiés dans } \\
\text { Nature et Science }\end{array}$ & $20 \%$ & 5 ans \\
\cline { 2 - 4 } & $\begin{array}{l}\text { Articles indexés dans } \\
\text { Science Citation Index } \\
\text { et Arts \& Humanities } \\
\text { Citation Index }\end{array}$ & $20 \%$ & 1 an \\
\hline Productivité & $\begin{array}{l}\text { Performance académique } \\
\text { taille de l'institution }\end{array}$ & $10 \%$ & \\
\hline
\end{tabular}

en compte des recherches menées dans les organismes beaucoup plus développés en Europe et notamment en France (le CNRS est au premier rang mondial pour les publications scientifiques) »... Tout ceci n'est que clabaudage. La ministre a oublié de préciser que le CNRS regroupe 11300 chercheurs et qu'il dispose d'un budget de 3,415 milliards d'euros, ce qu'aucune université au monde n'offre à ses chercheurs ! Quand on examine le Top 100, on s'aperçoit que l'Allemagne ne fait pas mieux que la France mais que 2 pays occidentaux beaucoup moins peuplés (le Canada et la Suisse) placent également 4 universités dans le Top 100 ! Peut-être existe-t-il des arguments bien plus irréfragables pour nous faire entrevoir la réalité.

Le classement de Shanghaï est établi à partir de 6 critères ( 5 seulement la première année et ils avaient tous la même valeur) obtenus par l'analyse de 4 domaines (Tab. I). La valeur des 2 premiers varient dans le temps. Par exemple, un prix Nobel ou une médaille Fields sont comptabilisés à $100 \%$ pour l'évaluation de la qualité de l'enseignement lorsqu'ils ont été reçus après 1990 et à $100 \%$ pour l'évaluation de la qualité de l'institution lorsqu'ils ont été reçus après 2000 . Les pourcentages diminuent avec l'ancienneté de la distinction et, dans les 2 cas, ils ne vieillissent pas de la même façon. Lorsque le lauréat est affilié à plusieurs institutions, il y a un partage. De même pour les publications, il y a une répartition en fonction des auteurs : $100 \%$ pour l'affiliation de l'auteur correspondant, $50 \%$ pour celle de premier auteur, $25 \%$ pour celle de l'auteur suivant et $10 \%$ pour celle des autres auteurs; à noter que l'on ne tient pas compte de l'impact factor de la revue. Enfin, la productivité est calculé en divisant le total des scores obtenus avec les 5 premiers critères par le nombre de chercheurs équivalent temps plein. Pour chaque critère, un score de 100 est attribué à la meilleure université et le score des autres universités est adapté en conséquence ; le score final est obtenu de la même façon. Sous une apparente rigueur, les auteurs du classement utilisent des indicateurs dont la pertinence n'a jamais été démontrée et ils éliminent le domaine productivité lorsqu'ils ne connaissent pas le nombre de chercheurs équivalent temps plein (seule donnée qui ne soit pas disponible sur internet)! De plus, ce classement se fait en l'absence de toute transparence et il n'est possible d'obtenir auprès des auteurs des précisions sur les données prises en compte pour évaluer ces différents critères. Sans doute en partie pour éviter d'être toujours assailli par les critiques ou les plaintes, le Professeur Liu a créé en 2009 un organisme, le Center for World-Class Universities, qui publie dorénavant le classement de Shanghaï. Il est ainsi plus difficile pour le ministre de l'Education chinois de relayer les plaintes.

Sans aller plus dans le détail, voilà déjà de quoi relativiser la pertinence de ce classement. Par ailleurs, on peut s'étonner qu'il ait été réalisé par un chimiste (formation initiale du Professeur Liu) ignorant tout sur l'évaluation de la recherche, de l'enseignement... d'où l'utilisation d'indicateurs simples et d'une méthode rudimentaire. Certes, le Professeur Liu a sans doute maintenant acquis des compétences dans ce domaine mais il reste prisonnier du système mis en place initialement. En 2009, Billaut et al. [2] ont réalisé une étude critique de ce classement avec la méthode MCDM (multiple-criteria decision making). Cette étude, très détaillée et bien structurée, comporte une partie technique difficilement abordable pour un néophyte. Après cette pérégrination dans une démarche méthodologique abstruse, on est convaincu que les indicateurs choisis par le Professeur Liu ne sont pas représentatifs et que la manipulation des chiffres pour attribuer un score de 100 pour la meilleure université modifie leur pertinence. Les auteurs résument ainsi leur analyse : «Our main conclusions are that the criteria that are used are not relevant, that the agrégation methodology is plagued by a number of major problems and that the whole exercise suffers from an insufficient attention paid to fundamental structuring issues ». En deux mots, ils reprochent au Professeur Lieu d'additionner des torchons et de serviettes qui, de plus, n'ont ni la même taille, ni la même couleur, ni la même qualité...

Le succès du classement de Sanghaï est difficilement compréhensible car plusieurs classements mondiaux, sans doute plus pertinents, sont disponibles: High Impact Universities, Performance Ranking of Scientific Papers for World Universities, QS World University Rankings, Webometrics Ranking of World Universities, Times Higher Education World University Rankings. Dans ces classements, l'ordre des universités est différent. Par exemple, Harward University n'est pas en première position et, pour la France, l'École Normale Supérieure de Paris et l'École Polytechnique (respectivement à la $71^{\mathrm{e}}$ place et entre la $201^{\mathrm{e}}$ et $300^{\mathrm{e}}$ dans le classement de Shanghaï 2013) sont placées bien avant l'Université Pierre et Marie Curie et l'Université Paris Sud. Le classement de Shanghaï n'a donc pas un caractère universel. Chaque université possède ses spécificités et il ne peut y avoir de modèle standard. Pour ceux qui auraient encore 
quelque sympathie pour le classement de Shanghaï ou pour ceux qui cherchent le contrepoison, Billaut et al. ont montré qu'en regroupant les 13 universités parisiennes en une seule entité, la nouvelle Université de Paris ferait jeu égal avec Harward University dans le classement de Shanghaï [2]. Cette solution qui ne demande aucun investissement supplémentaire, semble avoir été suggérée aux autorités politiques pour mettre un terme à leur émoi récurrent.

L'Union européenne tente de réagir. Elle a entrepris la réalisation d'un nouveau classement des 500 meilleures universités mondiales, U-Multirank [3], mais ce ne serait pas en fait un classement mais plutôt un outil interactif permettant de faire émerger les points forts (et les points faibles) de chaque institution et de les comparer entre elles; pour Laura Keustermans, conseillère politique à la League of European Research Universities, ce n'est pas un classement mais plutôt un « benchmark ». Lancé les 30 et 31 janvier 2013, à Dublin, ce classement veut prendre en compte 5 dimensions (l'enseignement, la recherche, le transfert de connaissance, l'internationalisation et l'implantation régionale), elles-mêmes subdivisées en une dizaine de critères. Il semble excessivement ambitieux et trop complexe. Initialement prévue pour 2013, la première version est attendue pour février 2014 mais il y a déjà eu de nombreuses critiques sur sa complexité et sa fiabilité (les renseignements devront être fournis par les universités). Les difficultés de réalisation et l'impossibilité de trouver un consensus ont favorisé l'apparition de tensions au sein du groupe en charge de la réalisation du projet. Certains comme la League of European Research Universities ont quitté le groupe et sont venus grossir les rangs des critiques impitoyables, dont quelques uns sont probablement des séides stipendiés de Thomson Reuters qui aimerait bien vulgariser son classement, le Times Higher Education World University Rankings. Le pronostic vital de UMultirank semble bien mal engagé, surtout si l'on se souvient qu'il s'agit d'un projet déjà ancien que l'on a ressuscité sous un autre nom. En effet, en 2008, sous la Présidence française, l'Union européenne avait pris l'initiative de réaliser un Ranking Europe's universities avec les mêmes objectifs [4]. Elle avait alors alloué 1,1 millions d'euros à ce projet qui devait être terminé fin mai 2011. Ce serait donc une divine surprise si le projet U-Multirank aboutissait. Et cela changerait-il réellement quelque chose?

\section{Références}

1. http://www.shanghairanking.com/ARWU2013.html ou http://www.arwu.org

2. Billaut J-C, Bouyssou D, Vincke P. Should you believe in the Shanghai ranking? An MCDM view. Scientometrics 2010;84: 237-63.

3. http://www.u-multirank

4. http://europa.eu/rapid/pressReleasesAction.do?reference=IP/ 08/1942\&format=HTML\&aged=0\&language $=E N \&$ guiLanguage $=e n$ 